\title{
Metodología para evaluar la exposición ocupacional a contaminantes químicos en altitud
}

\author{
Methodology to evaluate occupational exposure to \\ chemical contaminants at high altitude
}

\begin{abstract}
Margarita Zamora-Saa ${ }^{1}$ (D) 0000-0002-7415-4569
Jilberto Zamora-Saa ${ }^{2,3}$ (D) 0000-0002-5030-7516

${ }^{1}$ Escuela de Construcción, DUOC Universidad Católica, Santiago, Chile.

${ }^{2}$ Departamento de Ciencias Físicas, Universidad Andres Bello, Santiago, Chile.

${ }^{3}$ Millennium Institute for Subatomic physics at high energy frontier - SAPHIR, Santiago, Chile.
\end{abstract}

Fechas - Dates

Recibido: 2021.04.24

Aceptado: 2021.08.26

Publicado: 2021.10.15
Correspondencia · Corresponding Author

Margarita Zamora-Saa

mzamora@duoc.cl 


\title{
Resumen
}

Este artículo examina el actual método para evaluar la exposición a contaminantes químicos en altitud usando los Threshold Limit Value (TLV). Estos valores son establecidos para trabajos a nivel del mar en jornadas de 8 horas diarias y 40 horas semanales. Se analiza este problema aplicándolo a un grupo específico de mineros chilenos que trabaja sobre los 3000 m con jornadas excepcionales de 12 horas diarias. Se comparan dos alternativas para evaluar la exposición a contaminantes químicos: ajustes al TLV en altitud, y el cálculo de la dosis inhalada. Puesto que la ventilación por minuto es el parámetro fisiológico que cambia en altitud se propone y fundamenta como método de evaluación el cálculo de la dosis inhalada del contaminante químico.

Palabras clave: Valores límites; altitud; dosis inhalada.

\begin{abstract}
This study reviews the current method for assessing exposure to chemical contaminants at high altitude, which is based on the use of Threshold Limit Values (TLV). These TLVs were originally established for work performed at sea level, for 8-hour shifts over a 40-hour work week. Our study analyzes the problem for a specific group of Chilean miners working at altitudes above 3000, and for shifts of up to 12 hours a day. We examined different approaches for the evaluation of exposure to chemical contaminants: adjusting the TLV for work at high altitude and calculating inhaled dose. Since minute ventilation changes at different altitudes, we propose using calculated inhaled dose of the chemical contaminant as the method of choice.
\end{abstract}

Keywords: Threshold Limit Value; high altitude; inhaled dose.

\section{Introducción}

El 80\% de los yacimientos mineros en Chile está ubicado a más de 3000 metros sobre el nivel del mar ${ }^{(1)}$, los trabajadores de faenas mineras son aproximadamente $248.803^{(2)}$. En altitud disminuye la presión barométrica, y como resultado disminuye la presión parcial y la presión inspirada de oxígeno. Una de las primeras respuestas del organismo ante la hipoxia hipobárica es el aumento de la ventilación pulmonar $\left(V_{E}\right)^{(3)}$. En consecuencia, los trabajadores de altitud inhalan un volumen de aire mayor comparado con el que inhalarían a nivel del mar, esto implica una mayor exposición a los contaminantes químicos (CQ) presentes en los ambientes laborales de altitud.

Un problema importante para evaluar y controlar esta exposición es que no se cuenta con un valor límite umbral (Threshold Limit Value) TLV ajustado a la altitud. Los TLV para CQ son establecidos para condiciones normales, es decir, para trabajos a nivel del mar y con jornadas laborales de 8 horas diarias (hd). Un segundo problema es cuando en altitud se está expuesto a tóxicos con efectos aditivos; no se encuentra información que explique como evaluar la exposición combinada. 
La American Conference of Governmental Industrial Hygienists $(\mathrm{ACGIH})^{(4)}$ establece un criterio para evaluar exposiciones combinadas a $C Q$ a nivel del mar. Este criterio indica que cuando varios CQ ejerzan la misma acción sobre los mismos órganos o sistemas, sus efectos se considerarán aditivos, salvo que información adicional demuestre que son sinérgicos o independientes. Sin embargo, sobre exposición simultánea a dos o más CQ y a uno físico, como la presión barométrica baja debida a la altitud, hay pocos estudios que analicen el problema y propongan una solución para la evaluación. Estudios en animales expuestos a Monóxido de Carbono y altitud dan cuenta del efecto aditivo de estos riesgos ${ }^{(5)}$, y otros, sobre exposición a sílice y altitud confirman que en altitud se inhala una mayor dosis de sílice(6), pero no plantean como evaluar el efecto combinado. Este trabajo reporta la necesidad de contar con un método para evaluar la exposición a CQ en mineros chilenos que trabajan sobre 3000 metros por $12 \mathrm{hd}$. Se discuten algunas posibles soluciones.

\section{Resultados y Discusión}

El reglamento en Chile sobre condiciones sanitarias y ambientales básicas en los lugares de trabajo (Decreto 594/1999) propone un factor de corrección (Fa) para los TLV a partir de los 1000 m.s.n.m.(7):

$$
\mathrm{Fa}=P / 760
$$

donde:

$P$ : presión barométrica del lugar de trabajo.

El producto de Fa por el TLV, es el TLV ajustado para altitud. Con esta corrección se cumple el objetivo de reducir el valor del TLV en altitud. Hay pocos estudios que propongan ajustes para el TLV en altitud, en cambio, hay varias publicaciones sobre ajustes para un TLV en jornadas excepcionales ${ }^{\left({ }^{(}\right)}$.

Parece más confiable evaluar la exposición a CQ en altitud mediante la dosis inhalada del contaminante $\left(D_{i}\right)$, que ajustar el TLV:

$$
D_{i}=C_{c} \cdot V_{E} \cdot t_{E}
$$

donde:

$C_{c}$ : concentración del contaminante en $\mathrm{mg} / \mathrm{m}^{3}$

$t_{E}$ : tiempo de exposición en horas.

Debido a que $V_{E}$ es el parámetro respiratorio que cambia en altitud y que las jornadas de trabajo son de $12 \mathrm{hd}$, con esta metodología obtendríamos una mejor estimación de la exposición real del trabajador.

$V_{E}$ se puede medir o estimar mediante fórmulas. Medir $V_{E}$ en altitud no nos parece viable como práctica permanente, ya que, los tiempos de producción y el costo asociado son muy altos. Mediciones en altura fueron ya hechas por uno de los autores y publicadas ${ }^{(5)}$. Consecuentemente, proponemos calcular $V_{E}$. 
El trabajo realizado por Greenwald et a|(9) que considera más de 14000 datos, propone la siguiente relación para el cálculo de $V_{E}$ :

$$
V_{E}=e^{-8.57} \cdot f_{C}^{1.72} \cdot f_{R}^{0.611} \cdot E^{0.298} \cdot S^{-0.206} \cdot C_{V F}^{0.614}
$$

donde:

$\mathrm{f}_{c}$ : frecuencia cardiaca en latidos por minuto.

$f_{R}$ : frecuencia respiratoria en respiraciones por minuto.

E: edad en años,

$S$ : sexo, 1 para hombres y 2 para mujeres,

$C_{V F}:$ Capacidad vital forzada en litros.

Proponemos usar esta relación, ya que $C_{V F^{\prime}} f_{R^{\prime}}$ y $f_{C}$ darían cuenta de los cambios debidos a la altitud. Se sabe que a mayor cantidad de años trabajando en altitud mayor $C_{V F}{ }^{(5)}$.

Para el caso de los mineros chilenos el cálculo de la dosis con la fórmula citada es simple de realizar ya que estos valores están disponibles. En Chile, por normativa, se implementan programas de vigilancia médica y ambiental para trabajos sobre los 3000 metros. Estos programas incluyen exámenes médicos anuales y mediciones de la concentración ambiental de los contaminantes trimestralmente.

$D_{i}$ debiese ser comparada con una dosis máxima aceptable $\left(D_{\max }\right)$, donde la concentración es el TLV del CQ, usando $V_{E}$ para carga de trabajo media ${ }^{(10)}$ :

\section{Conclusiones}

Es necesario evaluar el comportamiento de la $C_{V F}$ en función de la altitud y años de exposición a esta junto con realizar estudios en la población chilena que permitan ajustar la fórmula de $V_{E}$ según altitud.

Dado que $V_{E}$ aumenta con la altitud, el mejor método de evaluación de la exposición a $C Q$ en altitud durante jornadas excepcionales es a través de la $D_{i}$. Comparándola con $D_{\text {max }}$ se podría estimar el riesgo y tomar las medidas de control adecuadas.

Para el caso de tóxicos aditivos se podría evaluar la exposición de la siguiente forma:

$$
\text { Dosis Aditiva }=\sum_{j=1}^{n}\left(\frac{D_{i}}{D_{\max }}\right)_{C Q_{j}}
$$

donde $C Q_{j}$ corresponde al contaminante químico j-ésimo.

La Dosis Aditiva no debiese ser superior a 1. 


\section{Agradecimientos}

El trabajo de Jilberto Zamora-Saa fue financiado por ANID - Millennium Science Initiative Program - ICN2019_044.

\section{Conflicto de intereses}

Declaramos no tener conflicto de intereses y no estar considerando otra revista para la publicación de este artículo.

\section{Bibliografía}

1. Departamento de Estudios de la Dirección del Trabajo, Ministerio del Trabajo, Gobierno de Chile. Una aproximación a las condiciones de trabajo en la gran minería de altura. 2011. 15 p. ISBN: 978-956-7978-11-3.

2. Servicio Nacional de Geología y Minería, Anuario de la Minería en Chile 2019. ISSN: 0066-5096. 2020. 186 p.

3. Vearrier D, Greenberg MI. Occupational health of miners at altitude: adverse health effects, toxic exposures, pre-placement screening, acclimatization, and worker surveillance. Clin Toxicol (Phila). 2011;49(7):629-40.

4. American Conference of Governmental Industrial Hygienists (ACGIH). Threshold limit values and biological exposure indices, Cincinnati, USA: Signature Publications; 2015.

5. McGrath JJ. Carbon monoxide studies at high altitude. Neurosci Biobehav Rev. 1988;12(3-4):311-4.

6. Bacaloni A, Zamora Saà MC, Sinibaldi F, Steffanina A, Insogna S. Respiratory parameters at varied altitudes in intermittent mining work. Int J Occup Med Environ Health. 2018;31(2):129-138.

7. Ministerio de Salud de Chile. Decreto Supremo 594: Reglamento sobre condiciones sanitarias y ambientales básicas en los lugares de trabajo. 29 Abril 2000.

8. Drolet D. Guide for the adjustment of permissible exposure values (PEVs) for unusual work schedules, 4th edition and updated. Institut de recherche Robert-Sauvé en santé et en sécurité du travail (IRSST); 2015. ISBN: 978-2-89631-798-1.

9. Greenwald R, Hayat MJ, Dons E, Giles L, Villar R, Jakovljevic DG, et al. Estimating minute ventilation and air pollution inhaled dose using heart rate, breath frequency, age, sex and forced vital capacity: A pooled-data analysis. PLoS One. 2019;14(7): e0218673.

10. Bacaloni A, Zamora Saà MC, Sinibaldi F, Steffanina A, Insogna S. Rischio contemporaneo di esposizione a silice cristallina aerodispersa ed elevata altitudine in minatori cileni: una valutazione non convenzionale. Proceedings of the II Congreso Internacional de Medicina del Trabajo Italia-Argentina, Rome, Italy, Nov 30 - Dec 2, 
2017; Imbriani M., De Sio S., Mormone M. et al, Eds.; Giornale Italiano di Medicina del Lavoro ed Ergonomia, Volume XXXIX, N.4, ISSN 1592-7830, Roma, 2017. 34-37. 